\title{
Future island universes in a background universe accelerated by a cosmological constant and by quintessence
}

\author{
Tzihong Chiueh and Xiao-Gang He \\ Department of Physics, National Taiwan University, Taipei, Taiwan 10764, Republic of China
}

(Received 26 December 2001; published 18 June 2002)

\begin{abstract}
We study bound object formation in a background universe accelerated by a cosmological constant and by quintessence. If the acceleration lasts forever, due to the existence of an event horizon, one would have naively expected the universe to approach a state of cold death. However, we find that many local regions in the universe can in fact be protected by their own gravity to form miniuniverses, provided that their present matter densities exceed some critical value. In the case with a cosmological constant ( $\Lambda$ cold dark matter cosmology), the condition for forming a miniuniverse is that the ratio of the present density parameters $\Omega_{i}^{0} / \Omega_{\Lambda}$ should be larger than a critical value 3.63. Such miniuniverses typically weigh less than $2 \times 10^{14}$ solar masses, with the lighter ones having tight and compact configurations. In the case with quintessence, the final ratio $\Omega_{i} / \Omega_{q}$ of a miniuniverse is found to be always larger than $w_{q}^{2}-w_{q}$, where $w_{q}$ is the equation-of-state parameter.
\end{abstract}

DOI: 10.1103/PhysRevD.65.123518

PACS number(s): 98.80.Es

\section{INTRODUCTION}

Recently direct evidence from the studies of the Hubble diagram for type-Ia supernovae [1] indicates that our universe is expanding at an increasing rate, i.e., it has accelerating expansion. This result compels one to seriously consider a dominant component of exotic dark energy, quintessence, in the cosmic energy balance. Accelerating expansion implies that the deceleration parameter $q=\left[\Omega_{m}\right.$ $\left.+\left(3 w_{q}+1\right) \Omega_{q}\right] / 2$ is negative, where $w_{q}$ is given by the cosmic equation of state, $p_{q}=w_{q} \rho_{q}$, of quintessence $Q$. As long as $w_{q}<-\frac{1}{3}$, the corresponding dark energy provides a negative contribution to $q$, and the relevant range of $w_{q}$ for acceleration lies in-between $-\frac{1}{3}$ and -1 . When $w_{q}$ assumes the extreme value -1 , constrained by the weak energy condition [2], the dark energy is reduced to the one arising from the cosmological constant $\Lambda$.

The acceleration may stop in the future, i.e., it may be a transient phenomenon with a time-dependent $w_{q}$, or it may last forever, depending on the nature of the dark energy [3]. A positive cosmological constant, consistent with the supernovae data at $z=1.7$ [4], leads to acceleration forever. The quintessence scenario with a constant $w_{q}$, not ruled out by data, can also lead to acceleration forever. There are profound implications for a forever-accelerating universe $[5,6]$. The universe will exhibit an event horizon; that is, there exist regions of the universe inaccessible to light probes. It has been argued that such a universe presents a challenge for string theories or any of the alternatives [5]. Many other implications of a cosmological constant and quintessence and specific models have been studied in the literature [7-9].

Naively speaking, in a forever-accelerating universe, two points at different spatial locations will, as measured at any one of the two points, eventually be pulled apart at the speed of light to approach the future horizon. Hence existing structures will be eternally frozen and the universe will approach a state of cold death. Is this an unavoidable consequence everywhere in the universe, or may some mechanisms be in operation that salvage the future? The present work confronts this issue and shows that the self-gravity of matter can protect a local universe from ending up with cold death, provided that the present local matter density is sufficiently high. Contrary to the above naive expectation, a large number of miniuniverses, including our own Local Group, will become self-bound and survive the destruction of cosmic repulsive force due to the quintessence. They can naturally arise despite the fact that these miniuniverses will still be falling into each other's horizon at a sufficiently late time and become isolated island universes.

At present, a variety of dark energy candidates can accommodate the experimental data. If the dark energy arises from a positive cosmological constant, the $\Lambda$ cold dark matter (CDM) cosmology, data from recent cosmic microwave background $(\mathrm{CMB})$ radiation $[10]$ further constrain the present energy density parameters to be $\Omega_{m}^{0}=0.35$ and $\Omega_{\Lambda}^{0}$ $=0.65$, while for quintessence, depending on the value $w_{q}$, the present matter density $\Omega_{m}^{0}$ and quintessence density $\Omega_{q}^{0}$ can be different [11].

For our purpose of addressing the future of local bound objects in an accelerating universe, the cosmological constant presents the worst scenario since the cosmological constant gives rise to the strongest repulsive force against formation of bound objects. If objects are not torn up by the cosmological constant repulsive force, they will survive in other scenarios.

The paper is arranged as follows. In Sec. II, we first consider the $\Lambda \mathrm{CDM}$ cosmology in detail, by assuming that the present universe is spatially flat with $65 \%$ energy given by the cosmological constant $\left(\Omega_{\Lambda}^{0}=0.65\right)$ and another $35 \%$ by the matter $\left(\Omega_{m}^{0}=0.35\right)$. In Sec. III, we then consider the case in which the cosmological constant is replaced by the quintessence with a constant $w_{q}$. In both cases, the miniuniverse can form by self-gravity, but the properties of critically bound miniuniverses are very different. Our conclusion is given in Sec. IV.

\section{BOUND OBJECT FORMATION WITH A COSMOLOGICAL CONSTANT}

We shall first adopt a toy model to address the questions of whether bound objects may still form after the background 
expansion has started to accelerate and how these objects become virialized. We then study a more realistic model where the observation or simulation-motivated features of virialized objects are taken into account.

\section{A. Self-binding by local gravity}

The toy evolutionary model for bound object formation assumes the universe consists of the background and an isolated cold dark matter fluctuation that grew from a small amplitude since the early epoch. The localized perturbation possesses spherical symmetry and consists of two concentric solid spheres of different densities. The inner sphere has a radius $r_{i}$ with a uniform dark-matter density $\rho_{i}$, which is greater than the background matter density $\rho_{m}$. The outer sphere has a radius $r_{\text {out }}$ and a uniform compensating underdensity, such that the averaged matter density within $r_{\text {out }}$ equals $\rho_{m}$. Any particle outside $r_{\text {out }}$ feels no extra gravity resulting from the overdensity and expands as it would have in a homogeneous universe. The inner sphere can be regarded as a closed Friedmann universe, which has a Hubble parameter, $H_{i}$, given by

$$
H_{i}^{2}=\left(\frac{\dot{r}_{i}}{r_{i}}\right)^{2}=\frac{8 \pi G}{3}\left(\rho_{i}+\rho_{\Lambda}\right)-\frac{\kappa_{i}}{r_{i}^{2}},
$$

where $\rho_{\Lambda}=(1 / 8 \pi G) \Lambda$ is a constant and $\kappa_{i}$ is the curvature of the miniuniverse.

A test particle at $r_{i}$ feels an effective potential $V\left(r_{i}\right)$,

$$
V\left(r_{i}\right)=-\frac{G M_{i}}{r_{i}}-\frac{4 \pi G}{3} \rho_{\Lambda} r_{i}^{2} .
$$

Conservation of matter yields a constant mass $M_{i}$ within $r_{i}$ with $M_{i}=4 \pi \rho_{i}\left(r_{i}\right) r_{i}^{3} / 3$. The potential $V(r)$ has a maximum at $r_{\max }$ determined by $V^{\prime}\left(r_{\max }\right)=0$ with $\quad V\left(r_{\max }\right)=$ $-4 \pi G \rho_{\Lambda} r_{\max }^{2}$ and $r_{\max }=\left[\left(\rho_{i}^{0} / 2 \rho_{\Lambda}\right)\right]^{1 / 3} r_{0}$, where the density at present has been denoted as $\rho_{i}^{0} \equiv \rho_{i}\left(r_{0}\right)$, with $r_{0}$ being the present radius of the overdense sphere. When the test particle reaches $r_{\max }$ with a zero velocity, it will be marginally bound. The density $\rho_{i}$ corresponding to this situation is called the critical binding density, denoted as $\rho_{i c}^{0}$ at present, and any local region with density larger than $\rho_{i c}^{0}$ will eventually turn around and collapse.

The curvature of the miniuniverse can be conveniently obtained at the turnaround, after which gravitational collapse ensues, by setting $\dot{r}_{i}^{2}=0$ and equating $-\kappa_{i} / 2$ to the potential at the turnaround. That is,

$$
\kappa_{i}=\frac{8 \pi G \rho_{\Lambda}}{3}\left(\frac{\rho_{i}\left(r_{0}\right)}{\rho_{\Lambda}} \frac{r_{0}^{3}}{r_{\mathrm{ta}}^{3}}+1\right) r_{\mathrm{ta}}^{2},
$$

where $r_{\mathrm{ta}}$ is the turnaround radius. In particular, for a critically bound miniuniverse, we have $r_{\mathrm{ta}}=r_{\max }$ and $\kappa_{i c}$ $=\kappa_{i}\left(r_{\max }\right)=8 \pi G \rho_{\Lambda} r_{\max }^{2}$.

The time span needed for the miniuniverse to evolve to the present epoch is given by

$$
\begin{aligned}
t_{i}= & \int_{0}^{r_{0}} \frac{d r_{i}}{r_{i} H_{i}} \\
= & \int_{0}^{r_{0}} \frac{d r_{i}}{r_{i} \sqrt{(8 \pi G / 3)\left(\rho_{i}+\rho_{\Lambda}\right)-\left(\kappa_{i} / r_{i}^{2}\right)}} \\
= & \frac{1}{\sqrt{6 \pi G \rho_{\Lambda}}} \\
& \times \int_{0}^{1} \frac{d y}{\sqrt{\left(\rho_{i}^{0} / \rho_{\Lambda}\right)+y^{2}-\left(3 \kappa_{i} / 8 \pi G \rho_{\Lambda} r_{0}^{2}\right) y^{2 / 3}}} .
\end{aligned}
$$

When $t_{i}$ is known, Eq. (4) can be inverted to solve for the present local density $\rho_{i}^{0}$. Thus one needs to obtain information about $t_{i}$. To this end we note that the miniuniverse should have started to grow from a small-amplitude perturbation at the beginning of matter domination around $z=3$ $\times 10^{3}$. Therefore, to a good approximation, the evolution time $t_{i}$ is equal to the background evolution time $t_{B}$. The time $t_{B}$ for the background Friedmann universe to reach the present ratio $\Omega_{m}^{0} / \Omega_{\Lambda}^{0}$ is

$$
\begin{aligned}
t_{B}=\int_{0}^{a_{0} \frac{d a}{\dot{a}}} & =\frac{1}{\sqrt{6 \pi G \rho_{\Lambda}}} \int_{0}^{1} \frac{d y}{\sqrt{\left(\Omega_{m}^{0} / \Omega_{\Lambda}^{0}\right)+y^{2}}} \\
& =\frac{1}{\sqrt{6 \pi G \rho_{\Lambda}}} \sinh ^{-1}\left(\sqrt{\frac{\Omega_{\Lambda}^{0}}{\Omega_{m}^{0}}}\right) .
\end{aligned}
$$

Setting $t_{i}=t_{B}$, one can determine the present density ratio $\rho_{i}^{0} / \rho_{\Lambda}$ for a given $\kappa_{i}$.

We consider three cases of interest for the discussions of the density ratio $\rho_{i} / \rho_{\Lambda}$ : (i) the critical miniuniverse, where the test particle reaches $r_{\max }$ with zero velocity and is therefore marginally bound; (ii) the case where the overdensity is larger than that of case (i) and the test particle has a zero velocity and turns around at present; (iii) the overdensity is even larger so that the turnaround occurred at the time when the background matter density $\rho_{m}^{\mathrm{eq}}$ equaled $\rho_{\Lambda}$, a particular epoch prior to which the matter in-fall had been vigorous.

For case (i), we have $\kappa_{i}=\kappa_{i c}=8 \pi G \rho_{\Lambda} r_{\max }^{2}$. Given the background density parameters $\Omega_{m}^{0}=0.35$ and $\Omega_{\Lambda}^{0}=0.65$, Eq. (4) along with Eq. (5) yield the present critical density ratio $\rho_{i c}^{0} / \rho_{\Lambda}=3.63\left[\Omega_{i c}\left(r_{0}\right)=2.36\right]$ and $r_{0}=r_{\max } / 1.22$.

We note that overdense regions with the present density parameter larger than the critical value $\Omega_{i c}\left(r_{0}\right)$ are abundant in the universe. In fact, nearly all future bound objects will never reach $r_{\max }$ with a turnaround radius $r_{\text {ta }}$ smaller than $r_{\max }$. Inserting $\kappa_{i}$ of Eq. (3) into Eq. (4), one obtains their present densities $\rho_{i}\left(r_{0}\right)$ for a given ratio $r_{\mathrm{ta}} / r_{0}$. For case (ii), the miniuniverse that is presently turning around, we have $r_{\mathrm{ta}}=r_{0}$, and it is found that $\rho_{i}^{0} / \rho_{\Lambda}=5.90$ with $\Omega_{i, 0}=3.83$. Any overdense region with a density greater than this value has already undergone collapse. For case (iii), it is found that $\rho_{i}^{\mathrm{eq}} / \rho_{\Lambda}=8.55$ with $\Omega_{i, \text { eq }}=5.52$.

Although the toy evolutionary model so far discussed may seem somewhat simplistic, the model nevertheless illustrates 
the essential physics [12] and supports our contention. From the above discussions, we clearly see that local bound objects can form against the cosmic repulsive force, as long as the local-density parameter $\Omega_{i}\left(r_{0}\right)$ at present is greater than 2.36. This value is considerably lower than the mean density parameter of the Local Group, which includes the Milky Way, the Andromeda galaxy, and more than a dozen smaller galaxies such as the Magellanic clouds. This suggests that the Local Group has defied the cosmic repulsive force and gravitational collapse is under way.

\section{B. Virialization}

Almost all bound objects, except for a set of measure-zero critically bound ones, will undergo, or have already undergone, gravitational collapse. It is instructive to examine further the issue of virialization after an object collapses. The virial density found here helps us to address deeper questions concerning the internal structures of bound objects to be addressed below. The virialized kinetic energy of a test particle is given by $\langle T\rangle=-\langle\vec{F} \cdot \vec{r}\rangle / 2$. For the simplified model under consideration, $\langle T\rangle$ is given by $\langle T\rangle=\left(G M_{i} / 2 r_{v}\right)$ $-\left(4 \pi G \rho_{\Lambda} r_{v}^{2} / 3\right)$, where $r_{v}$ is the virialized radius. This leads to

$$
-\frac{k_{i}}{2}=\langle T\rangle+\langle V\rangle=-\left(\frac{G M_{i}}{2 r_{v}}+\frac{8 \pi G}{3} \rho_{\Lambda} r_{v}^{2}\right) .
$$

From both energy and mass conservation together with the expression of $k_{i}$ at the turnaround, Eq. (3), with the subscript "0" replaced by "ta," one finds [13]

$$
\frac{\rho_{i, \mathrm{ta}}}{\rho_{\Lambda}}\left(2-\frac{r_{\mathrm{ta}}}{r_{v}}\right)+2\left(1-2 \frac{r_{v}^{2}}{r_{\mathrm{ta}}^{2}}\right)=0 .
$$

Regions with a space curvature $\kappa_{i}$ infinitesimally greater than $\kappa_{i c}$ of case (i) will finally collapse in the infinite future, and we shall also regard this situation as case (i) in a broad sense. It is straightforward to obtain that $r_{v} / r_{\mathrm{ta}}=1 / 2.73$ for case (i), since $\rho_{i, \text { ta }} / \rho_{\Lambda}=2$ and the corresponding $r_{v} / r_{0}$ is $1 / 2.24$. The ratio of the virialized density to the $\Lambda$ energy density is then found to be $\rho_{v} / \rho_{\Lambda}=41$. For cases (ii), we find $r_{v} / r_{0}=1 / 2.20$ and $\rho_{v} / \rho_{\Lambda}=62$. For case (iii), $r_{v} / r_{\text {ta }}$ $=1 / 2.13$ and $\rho_{v} / \rho_{\Lambda}=83$.

Case (iii) is interesting from the consideration that the matter in-fall was considerably vigorous prior to the turnaround but became suppressed afterward. Although its collapse started at $z=0.23$ when $\Omega_{m}=\Omega_{\Lambda}$, as the completion of collapse takes a time span twice the turnaround time, this object will be virialized in the future when the background universe reaches $\Omega_{\Lambda}=0.89$. By then the background scaling factor $a(t)$ becomes a factor 1.63 times the present value $a_{0}$.

After the bound object collapses and forms a virialized object, what does the bound object need to adjust so as to guard itself against the stripping repulsive force? We shall address this question by a model of somewhat more sophistication. It has been empirically found by cosmological $\mathrm{N}$-body simulations, which compute at best up to the present epoch, that cold dark matter particles tend to cluster into individual bound objects with a universal density profile. This density profile has been fitted by the empirical formula

$$
\rho=\frac{\Delta}{r\left(r_{s}+r\right)^{2}},
$$

where $\Delta$ and $r_{s}$ characterize the mass and inner scale length of the bound object [14]. This density profile has an $r^{-1}$ cusp and an $r^{-3}$ envelope extended out to the virial radius $r_{v}$. It is universal in that the parametrized functional form is independent of cosmological parameters and epochs, although the coefficients $\Delta$ and $r_{s}$ are not. Outside the virial radius $r_{v}$, the density profile must be truncated so that the interior matter can be blended into the background matter. Typically, the concentration parameter $r_{v} / r_{s}$ exceeds 5 for the present-day virialized objects, and the $r^{-3}$ envelope in the density profile is noticeable. Such a universal profile has been tested against observations favorably [15].

For our purposes, the universal profile can be extrapolated to describe the configurations of future bound objects. We will be concerned about how the concentration parameter $r_{v} / r_{s}$ and the characteristic density $\Delta$ vary with the boundobject formation time. Unfortunately, the virial radius $r_{v}$ is undefined in Eq. (8), therefore prohibiting us from using this density profile directly. One hence needs to seek another method to address the evolution of the universal profile.

The following strategy may be adopted for a quantitative evaluation of the evolution of the profile. The steady-state collisionless dark matter is described by the phase-space distribution function $f\left(C_{1}(\mathbf{x}, \mathbf{p}), C_{2}(\mathbf{x}, \mathbf{p}), \ldots\right)$, where $C_{i}$ 's are the constants of motion. For convenience, we assume the momentum $\mathbf{p}$ to be isotropic and the mass density spherically symmetric. It follows that $f\left(C_{i}\right)=f(E)$, a function of only particle energy $E$. Set $f(E)=0$ for unbound particles that have $E>0$. Upon integrating out the momentum from $f(E)\left[=f\left(p^{2} / 2 m+\phi\right)\right]$, we obtain the mass density $\rho(\phi)$, a function of the potential $\phi$ of all forces. The universal profile Eq. (8) is obtained from the Poisson equation for an appropriate $\rho(\phi)$. The functional form of $\rho(\phi)$ can be approximated by the following considerations. The inner singular density profile requires $\rho(\phi) \sim\left(\phi-\phi_{\min }\right)$ near the potential minimum $\phi_{\min }$ at $r=0$. On the other hand, the outer $r^{-3}$ behavior requires $\rho(\phi) \sim-\phi^{3}$ at the edge of the potential well, $\phi \rightarrow 0$, where the bound particles are about to escape. Thus these desired behaviors can be captured by

$$
\rho(\phi)=-\frac{\alpha \phi^{3}}{\exp \left[\beta\left(\phi-\phi_{\min }\right)\right]-1} .
$$

Normalizing all quantities in dimensionless form, we arrive at the Poisson equation,

$$
\frac{1}{x^{2}} \frac{d}{d x}\left(x^{2} \frac{d \psi}{d x}\right)+1=-\frac{g \psi^{3}}{\exp [b(\psi+1)]-1},
$$

where $\psi \equiv \phi /\left|\phi_{\min }\right|(\leqslant 0), x \equiv r / L$ with the characteristic length $L \equiv \sqrt{\left|\phi_{\min }\right| / 8 \pi G \rho_{\Lambda}}, \quad b \equiv \beta\left|\phi_{\min }\right|, \quad$ and $\quad g$ 


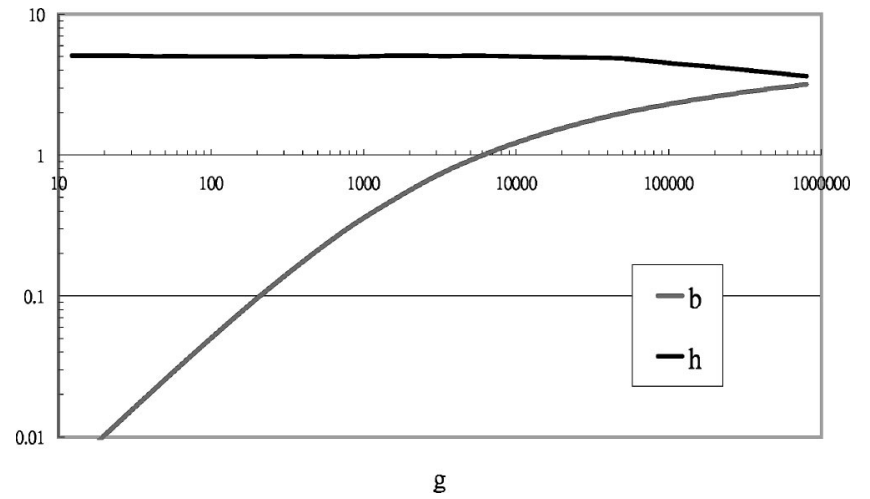

FIG. 1. Nonlinear eigenvalues $(g, b)$ for Eq. (10). When $b$ $<0.1$, the solutions are in a similarity scaling regime. Also shown is the half-mass weighted radius, $h$. The constancy of $h$ implies that the $r^{-1}$ core contains more than half of the total mass.

$\equiv \alpha\left|\phi_{\min }\right|^{3} / 2 \rho_{\Lambda}$. The gravitational potential $\phi_{g}$ on the lefthand side of the Poisson equation has been replaced by $\phi$ $+(4 \pi G / 3) \rho_{\Lambda} r^{2}$.

The solutions to Eq. (10) require numerical integration. Different values of $g$ and $b$ yield different $\Delta$ and $r_{s}$ of Eq. (8). This is a nonlinear eigenvalue problem, and there exists a locus in the eigenvalue $(g, b)$ space, along which the bound objects satisfy the desired boundary condition that $\rho$ vanishes at the potential maximum. Figure 1 presents the parameter $b$ as a function of $g$ for these bound objects. It is instructive to seek a measure for the inner $r^{-1}$ core region. Also plotted in Fig. (1) is the dimensionless half-mass-weighted radius $h \equiv\langle x D\rangle_{1 / 2}$ of these virialized objects, where $D$ $\equiv \rho / 2 \rho_{\Lambda}$. The quantity $\langle x D\rangle_{1 / 2} \equiv 3 \int_{0}^{x_{1 / 2}} x^{3} D d x / x_{1 / 2}^{3}$, and $x_{1 / 2}$ is defined to satisfy $\int_{0}^{x_{1 / 2}} x^{3} D d x \equiv(1 / 2) \int_{0}^{x_{\max }} x^{3} D d x$ with $D\left(x_{\max }\right)=0$. Note that the parameter $g$ varies by a factor of $10^{5}$ and $b$ by several hundred, and yet the quantity $h$ does not change in most parameter space until near the high end of $(g, b)$, where it decreases only by $50 \%$. This result shows that the $r^{-1}$ core contains more than half of the mass for most solutions, except for those with $g \geqslant 5 \times 10^{4}$.

Plotted in Fig. 2 are the density and potential profiles of three solutions with $(g, b)=(12,0.0063),\left(5 \times 10^{4}, 2\right)$, and

Density and Potential Profiles

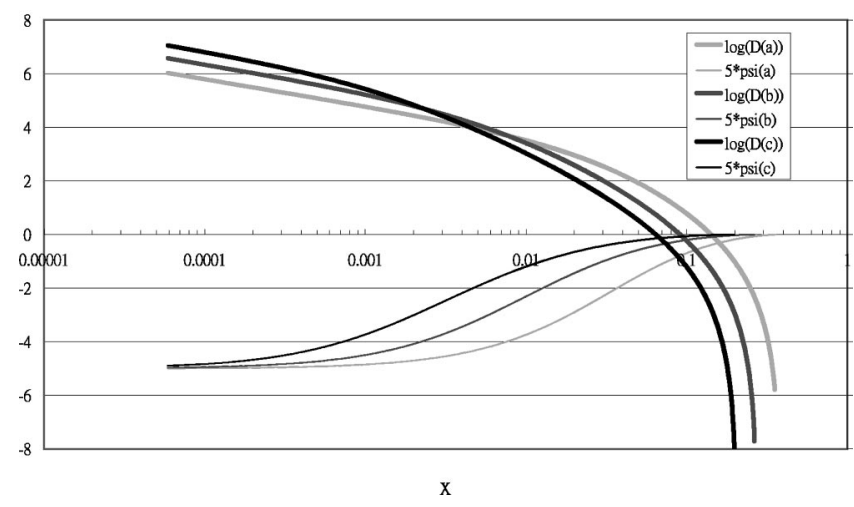

FIG. 2. Dimensionless densities $D$ (in log scale) and potentials $\psi$ for solutions (a), (b), and (c) of Eq. (10). $\left(8 \times 10^{5}, 3.2\right)$. These solutions, denoted as (a), (b), and (c), respectively, have progressively stronger cores of smaller sizes as both $g$ and $b$ increase. Solution (a), having an extended $r^{-1}$ core, gives no detectable outer envelope in the density profile, whereas solution (c), with a strong and compact core, contains a distinct $r^{-3}$ envelope. These solutions are expected to have progressively larger ratios of $\rho_{v} / \rho_{\Lambda}$, corresponding to virialized objects formed at progressively earlier epochs.

As virialized objects formed at different epochs appear differently, a future observer ought to be able to date these objects by morphology. To determine the formation epochs, we first start from the extended-core solution (a), which is at the small end of the $(g, b)$ spectrum shown in Fig. 1. It has already been well within a self-similar scaling regime beginning around $(g, b) \sim(196,0.1)$, where the denominator of $\rho(\phi)$ in Eq. (9) can be expanded to the leading order of small $\beta$, yielding $g / b \rightarrow 1953$. Different solutions in the scaling regime have the same profiles and hence solution (a) can be identified to be the virialized configuration of the last collapsed objects. Making use of the result derived earlier that $\rho_{v} / \rho_{\Lambda}=41$ within the virial radius $x_{v}$ for the last bound object, we determine the mass fraction, equal to 0.972 , within the virial radius.

Assuming that the mass fractions, 0.972, within the virial radii of all solutions remain the same, we find that $\rho_{v} / \rho_{\Lambda}$ $=65$ and 85 for solutions (b) and (c), respectively. According to the virialization model analyzed earlier, solutions (b) and (c) correspond to the virialized objects with turnaround times at $z=0$ and $z=0.23$, or cases (ii) and (iii) considered earlier, and they are to complete the collapse in the future when the scaling factors $a / a_{0}$ become 2.25 and 1.63 , respectively.

We note that the redshift evolution of morphology can be rather rapid for these future virialized objects formed in between $a / a_{0} \sim 1.5$ and 4.5 ; in contrast, the collapsed objects up to the present do not show much difference in morphology, all having a compact core and an $r^{-3}$ envelope.

Common to all virialized objects shown in Fig. 2 is that matter is mostly confined at a considerable distance away from the vacuum radius $x_{\max }$. Moreover, as the potential $\psi$ vanishes quadratically $\left(x-x_{\max }\right)^{2}$ near the potential maximum, the density should vanish as $\left(x-x_{\max }\right)^{6}$, according to Eq. (9). Using the force balance of pressure against gravity, it means that the local temperature vanishes as $\left(x-x_{\max }\right)^{2}$ at the edge. That is, the dark matter is cold at the outer edge of the miniuniverse. Moreover, conservation of the phase-space volume for collisionless particles demands that $\Delta r^{3} \Delta v^{3}$ remains a constant, and when $\Delta v^{3} \rightarrow 0$ we have $\Delta r \rightarrow \infty$. That is, the miniuniverse contains a thick outer layer of cold dark matter particles.

How do these cold particles result? Some fraction of collapsed dark-matter particles must have been pulled away by the cosmic repulsive force to the background during virialization. Thus, evaporation should serve as an important cooling mechanism to produce a thick outer layer of cold particles. The mass loss due to evaporation may be estimated. We note from Fig. 2 that the cool matter outside the virial radius $r_{v}$ occupies about $90 \%$ of the volume enclosed by $x_{\max }$, but it amounts to only $2.8 \%$ of the total mass. The 
evaporated material cannot be much more than the cool matter located in the thick outer layer. Therefore, the small mass loss should not create any problem for the above quantitative analyses that assume mass conservation during virialization.

Finally, the typical masses of bound objects formed at various epochs are also of relevance in understanding the future world. As the linear power spectrum $P(k)$ is well understood [16,17], this issue can be properly addressed as well. With a given conserved $\kappa_{i}$, the radius $r_{i}$ can be traced back to a sufficiently high $z$ through Eqs. (4) and (5), thereby fixing the amplitude of linear overdensity by the relation $\delta \rho(z)=3 \kappa_{i} / 8 \pi G r_{i}^{2}(z)$. On the other hand, $\delta \rho(z)$ is also given by $g(z) \int_{0}^{\pi / r_{i}(z)} 4 \pi k^{2} P(k) d k$, where $g(z)$ is the growth factor of linear perturbations [18]. Identification of the two $\delta \rho$ 's allows $r_{i}(z)$ to be determined, and the enclosed mass is simply $M=4 \pi \rho(z) r_{i}^{3}(z) / 3$, a redshift-independent quantity, as it should be. The typical mass of the last bound objects [solution (a) of Fig. 2] is found to be $2 \times 10^{14}$ solar masses, the mass of a poor galaxy cluster, and those for solution (b) and (c) are $10^{14}$ and $4.5 \times 10^{13}$ solar masses, respectively (assuming $H_{0}=65 \mathrm{~km} / \mathrm{s} / \mathrm{Mpc}$ ).

\section{BOUND OBJECT FORMATION WITH QUINTESSENCE}

In this section, we study the condition for bound object formation under the repulsive force of the quintessence with a constant $w_{q}$. At present, experimental data allow for the possibility that the accelerating cosmic expansion is due to quintessence with a relatively wide range of constant $w_{q}(\geqslant$ $-1)$ and energy densities [11].

In order to have an accelerating universe at present, the equation-of-state parameter $w_{q}$ should satisfy

$$
w_{q}<-\frac{1}{3}\left(1+\frac{\Omega_{m}^{0}}{\Omega_{q}^{0}}\right) \text {. }
$$

Unfortunately, the value of $\Omega_{m}^{0} / \Omega_{q}^{0}$ is still rather uncertain, and its plausible value deduced from the data correlates with $w_{q}$, with a larger $\Omega_{m}^{0} / \Omega_{q}^{0}$ for a smaller $w_{q}$ in a flat universe. For example, when $\Omega_{m}^{0}=35 \%$ and $\Omega_{q}^{0}=65 \%$ to make up the energy budget for a flat universe, Eq. (11) implies $w_{q}<$ -0.5 , but the existing data push the plausible $w_{q}$ to be near -1 . Nevertheless, to have an idea as to how the results vary with $w_{q}$, we shall consider three representative values, $w_{q}$ $=-0.5,-0.75$ and -1 , in our later discussions.

The quintessence energy density varies with the scaling factor as $\rho_{q}=\rho_{q}^{0}\left(a / a_{0}\right)^{-3\left(1+w_{q}\right)}$. The reference background cosmological time $t_{B}$ in this case is given by

$$
t_{B}=\frac{1}{\sqrt{6 \pi G \rho_{q}^{0}}} \int_{0}^{1} \frac{d y}{\sqrt{\Omega_{m}^{0} / \Omega_{q}^{0}+y^{-2 w_{q}}}} .
$$

For the study of bound miniuniverses, the situation becomes more complicated than that with a cosmological constant because the space curvature $\kappa_{i}$ within the collapse region is time-dependent, and Eq. (4) is no longer valid. (The analysis presented in Ref. [19], which assumes a constant $\kappa_{i}$, is hence invalid.) This problem should be analyzed using the momentum component of the Einstein equation, which describes the force balance of inertia with gravity and quintessence force. It can be understood as follows. Since the self-gravity of quintessence is negligible, the repulsive force of quintessence at a radius $r$ varies with time as $\left[a_{0} / a(t)\right]^{3\left(1+w_{q}\right)}$. This is a time-dependent force, thereby leading to a time-dependent space curvature.

The momentum component of the Einstein equation is a second-order differential equation:

$$
\frac{\ddot{r}}{r}=-\frac{4 \pi G}{3}\left[\rho_{i}+\left(1+3 w_{q}\right) \rho_{q}\right] .
$$

Defining $x=\left(a / a_{0}\right), y=\left(r / r_{0}\right)$, one has

$$
\begin{aligned}
& \dot{x}=\sqrt{\frac{8 \pi G}{3} \rho_{q}^{0}} \sqrt{\left(\Omega_{m}^{0} / \Omega_{q}^{0}\right)(1 / x)+x^{-\left(1+3 w_{q}\right)}}, \\
& \ddot{y}=-\frac{4 \pi G}{3} \rho_{q}^{0} y\left[\frac{\Omega_{i}^{0}}{\Omega_{q}^{0}} y^{-3}+\left(1+3 w_{q}\right) x^{-3\left(1+w_{q}\right)}\right] .
\end{aligned}
$$

Combining these two equations, one arrives at

$$
\begin{aligned}
& 2 \bar{y}^{\prime \prime} \bar{x}\left(1+\bar{x}^{\left.-3 w_{q}\right)=}=\bar{y}^{\prime}\left[1+\left(1+3 w_{q}\right) \bar{x}^{-3 w_{q}}\right]\right. \\
&-\left(\frac{\bar{x}^{2}}{\bar{y}^{2}}+\left(1+3 w_{q}\right) \frac{\bar{y}}{\bar{x}} \bar{x}^{-3 w_{q}}\right),
\end{aligned}
$$

where $\bar{x} \equiv x\left(\Omega_{m}^{0} / \Omega_{q}^{0}\right)^{1 / 3 w_{q}}, \bar{y} \equiv y\left(\Omega_{m}^{0} / \Omega_{q}^{0}\right)^{1 / 3 w_{q}}\left(\Omega_{m}^{0} / \Omega_{i}^{0}\right)$, and $\bar{y}^{\prime}=d \bar{y} / d \bar{x}$.

We may solve Eq. (15) to obtain the conditions for bound object formation at any background cosmic time $t_{B}$, cf., Eq. (12). However, due to the wide range of possible parameters for the quintessence cosmology, we shall instead concentrate on the critically bound miniuniverse. The asymptotic state of the critical miniuniverse can be found by letting both $\bar{y} \rightarrow \infty$ and $\bar{x} \rightarrow \infty$ in the infinite future. The existence of such a state also requires that the background expansion be accelerating, i.e., $w_{q}<-\frac{1}{3}$. It then follows that Eq. (15) has a scaling solution, $\bar{y}=\left(\Omega_{i \infty} / \Omega_{q \infty}\right)^{1 / 3} \bar{x}^{\left(1+w_{q}\right)}$, where

$$
\frac{\Omega_{i \infty}}{\Omega_{q \infty}}=w_{q}^{2}-w_{q},
$$

by matching the coefficients in Eq. (15). The subscript " $\infty$ " refers to an evaluation of a quantity at a reference epoch in the infinite future.

The space curvature $\kappa_{i}$ for this miniuniverse defined as minus twice the sum of the kinetic energy and the potential energy [of the force used in Eq. (14)] is given, in the asymptotic limit, by

$$
\kappa_{i}=-\left(\frac{a}{a_{\infty}}\right)^{-\left(1+w_{q}\right)}\left(1+3 w_{q}\right) 4 \pi G \rho_{q \infty} r_{\infty}^{2},
$$




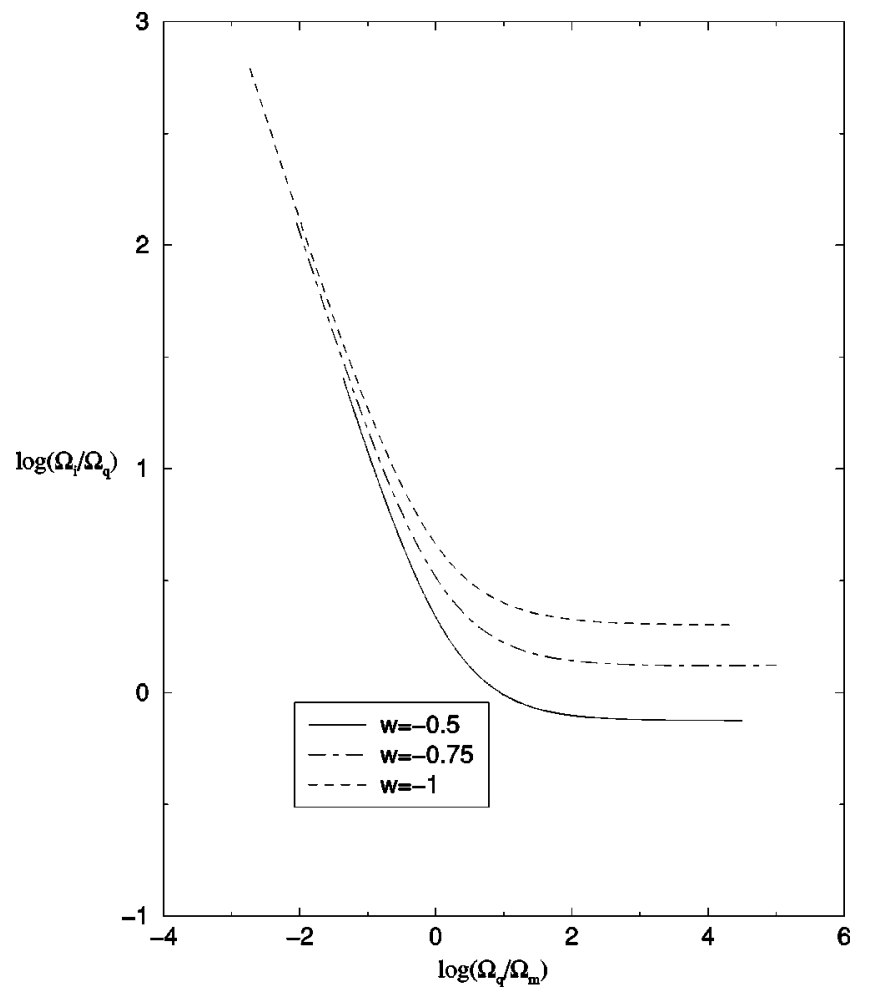

FIG. 3. The barely bound miniuniverse critical density as a function of time. The vertical axis is $\log \left(\Omega_{i} / \Omega_{q}\right)$ and the horizontal axis is $\log \left(\Omega_{q} / \Omega_{m}\right)$.

where Eq. (16) has been used. This confirms the statement made earlier that with quintessence the curvature is timedependent.

The space curvature decreases with time as $a^{-\left(1+w_{q}\right)}$ but its magnitude cannot be determined from the scaling solution; it can be fixed only by integrating Eq. (15) to obtain the entire solution. In this asymptotic state, the absolute values of kinetic, potential, and total energies all have the same time dependence with comparable magnitudes. For $w_{q}=-1$, we recover the cosmological constant case with $\rho_{i \infty} / \rho_{q \infty}=2$ and $\kappa_{i}$ given in Eq. (3). The above critical miniuniverse is very different from that in the case with a cosmological constant, in that its size grows indefinitely since $r$ grows as $a^{\left(1+w_{q}\right)}$.

One may extrapolate further the ratio $\rho_{i \infty} / \rho_{q \infty}$ back to the present by integrating Eq. (15) numerically. The results are shown in Fig. 3. In Fig. 3 we show $\log \left(\Omega_{i} / \Omega_{q}\right)$ as a function of $\log \left(\Omega_{q} / \Omega_{m}\right)$ for $w_{q}=-0.5,-0.75$, and -1 . From Fig. 3, one can read off the densities of the critically bound miniuniverse on the vertical axis for various background densities on the horizontal axis. [The horizontal axis can be regarded as another way to express the background cosmic time, cf., Eq. (12).] Regions whose present local densities exceed by a finite amount a given curve shown in Fig. 3 will turn around within a finite time and become virialized in a manner similar to that discussed in Sec. II, though the details differ [20].

However, it is interesting to note that there are differences for the virialized objects between the general quintessence cosmology and the $\Lambda \mathrm{CDM}$ cosmology. For the case with quintessence, a local region with an energy infinitesimally smaller than the critically bound condition (local matter den- sity larger than the critical density by an infinitesimal amount), the region will undergo collapse in the infinite future. The bound object has a nearly zero binding energy, as can be seen from Eq. (17), and hence after virialization its virial radius $r_{v}$ discussed in Sec. II will be infinitely large, a great contrast to the $w_{q}=-1$ case where the just bound objects have finite sizes.

As has been mentioned earlier in this section, the precise value of $\Omega_{m}^{0} / \Omega_{q}^{0}$ is yet to be determined and the plausible value of $\Omega_{m}^{0} / \Omega_{q}^{0}$ in fact anticorrelates with the value of $w_{q}$. Though for $w_{q}=-1$ the most plausible $\Omega_{m}^{0} / \Omega_{q}^{0}=0.35 / 0.65$, for $w_{q}=-0.75$ and -0.5 the most plausible $\Omega_{m}^{0} / \Omega_{q}^{0}$ decreases to approximately $0.1 / 0.9$ and $(\approx) 0 / 1$, respectively [11]. Precise knowledge for the content of energy forms, which can be acquired from more accurate calibration of type-Ia supernovae and measurements of $\mathrm{CMB}$ radiation as well as other observations [11,21], is crucial for making good use of Fig. 3.

\section{DISCUSSIONS AND CONCLUSIONS}

In this work, we have quantitatively shown that miniuniverses can form against the repulsive force of a cosmological constant or quintessence with a constant equation-of-state parameter $w_{q}$, as long as they have sufficiently high densities at present. These miniuniverses, when virialized, have distinctly different configurations depending on the formation time. Bound objects that form earlier appear more compact and are confined by deeper gravitational potentials. This issue is addressed in detail for the $\Lambda \mathrm{CDM}$ cosmology, where the miniuniverses are found to be typically no heavier than a poor galaxy cluster.

As discussed in Sec. II, the actual size of the miniuniverse varies, depending on the primordial density power spectrum. It is instructive to examine miniuniverses of very large scale. We note that a marginally bound miniuniverse has a gravitational radius $r_{g}^{2}=c^{2} r_{\text {max }}^{2} / \kappa_{i}=c^{2} /\left(3 \Omega_{\Lambda} H_{0}^{2}\right)$ in the case of $\Lambda$ cosmology. If a marginally bound miniuniverse has a physical size larger than $r_{g}$, no light emitted within can escape from it, and hence it behaves like a black hole when observed from the exterior. Such a miniuniverse is absolutely stable against the background pulling force. We therefore obtain the minimal size $r_{\text {min }}$ of an absolutely stable and marginally bound miniuniverse to be $c / \sqrt{3 \Omega_{\Lambda}} H_{0}$, which is comparable to the Hubble radius of the background universe.

For miniuniverses with larger local densities, the required radius is smaller. These miniuniverses will eventually undergo catastrophic collapse, and can asymptotically be described by a static space-time metric - the Schwarzschild-de Sitter metric:

$$
\begin{aligned}
d s^{2}= & \left(1-\frac{2 G M}{r}-\frac{\Lambda}{3} r^{2}\right) d^{2} t \\
& -\left(\frac{d^{2} r}{1-2 G M / r-\Lambda r^{2} / 3}+r^{2} d^{2} \theta\right. \\
& \left.+r^{2} \sin ^{2} \theta d^{2} \phi\right)
\end{aligned}
$$


It has two horizons: an inner horizon at a modified Schwarzschild radius and an outer horizon pertaining to the accelerating cosmic expansion. The region in between the two horizons is unstable, in that an observer located in between the two horizons must eventually fall onto either of them.

Our local universe cannot be within such a closed universe, as the angular scale of the first peak in the CMB anisotropy power spectrum indicates the local universe within the Hubble radius to be spatially flat [10]. This, however, does not preclude the possibility that regions outside the present Hubble horizon may have sufficiently large superhorizon overdensities to become a closed universe. The required present matter overdensity $\Omega_{i}^{0} / \Omega_{m}^{0}$ in such universes is as high as 6.75 . These regions are very rare if the primordial density fluctuation is indeed scale-invariant, created during the inflation in the early universe, with a power spectrum linearly proportional to the wave number $k$. Such local closed universes should be more abundant in the past, and some of them may have been within our present horizon, perhaps in the form of super massive black holes.

In the case of quintessence, the situation is more complicated because the miniuniverse has a time-dependent space curvature. The curvature actually decreases with time, due to the fact that matter and quintessence as a whole can be regarded as a nonideal fluid and the local matter overdensity gives rise to entropy fluctuations. Space curvature fluctuations pertinent to entropy fluctuations always decrease monotonically in time, regardless of whether the fluctuations are of subhorizon size or superhorizon size. However, adiabatic superhorizon fluctuations can also give rise to curvature fluctuations with no time dependence, for which quintessence fluctuations can grow and collapse as well. They are more relevant for the discussions of the local closed universe in the context of quintessence cosmology. Unfortunately, our analysis presented in Sec. III is confined to entropy fluctuations, where the quintessence fluctuation is negligible. Nevertheless, the dynamics of such a local closed universe with a constant space curvature is relatively simple and like that of a homogeneous universe. Thus the discussion given in the preceding paragraph for $\Lambda$ cosmology also holds for quintessence cosmology. The supermassive black hole so formed should contain a substantial amount of dense quintessence field within the Schwarzschild radius, and its asymptotic space-time metric depends on the yet unknown nature of the quintessence field.

Let us return to the miniuniverses arising from entropy fluctuations and of subhorizon size analyzed in Sec. III. As long as the matter density is larger than the critical density given in Fig. 3 for the corresponding equation-of-state parameter, the local region will be dominated by the matter gravity and collapses to become a miniuniverse. These miniuniverses will become virialized in a manner similar to those of the $\Lambda \mathrm{CDM}$ cosmology [20], due to the fact that virialization depends mainly on the balance between gravity and pressure, and to a lesser degree on the large-scale cosmic pulling force. Despite the similarity, the details can be different. For example, conservation of total energy within the bound object, which is essential for applying the virial condition, no longer holds in quintessence cosmology, and hence the resulting virial overdensity within the object deviates from that of the $\Lambda \mathrm{CDM}$ cosmology. Also the marginally bound miniuniverse has an infinitely large virial radius.

At any rate, a single tightly bound miniuniverse will, in the future, emerge from the Local Group, whose appearance resembles solution (c) of Fig. 2 in the case of $\Lambda$ CDM cosmology. Interior to the miniuniverse, local physics in the future will remain the same as what we experience presently. However, the miniuniverses will become isolated island universes in the future where no communication among them is possible, and each is a lone world. When observed within a given miniuniverse, all other miniuniverses originally within the horizon will eventually fall onto the horizon and become frozen. Lights emitted from the horizon are frozen as well, making other island universes dark and invisible.

The detailed properties of the miniuniverses depend on whether the accelerating expansion is due to the cosmological constant or quintessence. New experimental data from various observations in the coming decades are expected to reveal more information. By then we will have a better understanding of the properties of miniuniverses. If the acceleration of our universe at present is indeed due to a cosmological constant or quintessence with constant $w_{q}$, our universe will enter a new era, the era where all island universes are falling onto each other's horizon and appear to fade away. It is, nevertheless, comforting to know that the Milky Way galaxy is in one of these island universes, within each of which most physics remains the same as it is today. Review of the details of how an island universe will eventually end itself with the physics known today can be found in Ref. [22].

\section{ACKNOWLEDGMENTS}

This work is supported in part by National Science Council under Grants No. NSC 89-2112-M-002-058 and No. NSC 89-2112-M-002-065, and in part by the Ministry of Education Academic Excellence Project 89-N-FA01-1-4-3.
[1] S. Perlmutter et al., Nature (London) 391, 51 (1998); Astrophys. J. 517, 565 (1999); A.G. Riess et al., Astron. J. 116, 1009 (1998).

[2] S. Hawking and G. Ellis, The Large Scale Structure of SpaceTime (Cambridge University Press, Cambridge, England, 1973).
[3] J. Barrow, R. Bean, and J. Magueijo, Mon. Not. R. Astron. Soc. 316, L41 (2000); A. Albrecht and C. Skordis, Phys. Rev. Lett. 84, 2076 (2000); S.M. Carroll, ibid. 81, 3067 (1998); E. Halyo, J. High Energy Phys. 10, 025 (2001); J. Cline, ibid. 08, 035 (2001).

[4] A.G. Riess et al., Astrophys. J. 560, 49 (2001). 
[5] S. Hellerman, N. Kaloper, and L. Susskind, J. High Energy Phys. 06, 003 (2001); W. Fischler et al., ibid. 07, 003 (2001).

[6] X.-G. He, astro-ph/0105005; J. Moffat, hep-th/0105017; J.-A. Gu and W.-Y. Hwang, astro-ph/0106387.

[7] R.R. Caldwell, R. Dave, and P.J. Steinhardt, Phys. Rev. Lett. 80, 1582 (1998); I. Zlatev, L. Wang, and P.J. Steinhardt, ibid. 82, 896 (1999); P.J. Steinhardt, L. Wang, and I. Zlatev, Phys. Rev. D 59, 123504 (1999).

[8] Y. Fujii, Phys. Rev. D 26, 2580 (1982); L.H. Ford, ibid. 35, 2339 (1987); P.J. Peebles and B. Ratra, Astrophys. J. Lett. 325, L17 (1988); Y. Fujii and T. Nishioka, Phys. Rev. D 42, 361 (1990); K. Sato, N. Terasawa, and J. Yokoyama, in Proceedings of the XXIVth Recontre de Moriond, The Quest for the Fundamental Constants in Cosmology, edited by J. Audouze and J. Tran Thanh Van (Editions Frontières, France, 1990), p. 193; T. Nishioka and S. Wada, Int. J. Mod. Phys. A 8, 3933 (1993).

[9] N. Arkani-Hamed, L.J. Hall, C. Kolda, and H. Murayama, Phys. Rev. Lett. 85, 4434 (2000); S.M. Barr and D. Seckel, Phys. Rev. D 64, 123513 (2001); T. Chiueh, ibid. 65, 123502 (2002).

[10] Boomerang Collaboration, P. de Bernardis et al., Nature (London) 404, 955 (2000); Maxima Collaboration, S. Hanany et al., Astrophys. J. Lett. 545, L1 (2000).
[11] See, for example, M. Signore and D. Puy, astro-ph/0108515; P. Binetray, Int. J. Theor. Phys. 39, 1859 (2000).

[12] J.A. Peacock, Cosmological Physics (Cambridge University Press, Cambridge, England, 1999); A.R. Liddle and D. Lyth, Cosmological Inflation and Large-Scale Structures (Cambridge University Press, Cambridge, England, 2000).

[13] O. Lahav, P.B. Lilje, J.R. Primack, and M.J. Rees, Mon. Not. R. Astron. Soc. 251, 128 (1991).

[14] J.F. Navarro, C.S. Frenk, and S.D.M. White, Astrophys. J. 462, 563 (1996); 490, 493 (1997).

[15] J.A. Tyson, G.P. Kochanski, and I.P. dell'Antonio, Astrophys. J. Lett. 498, L107 (1998); L.L.R. Williams, J.R. Navarro, and M. Bartelmann, Astrophys. J. 527, 535 (1999).

[16] G. Efstathiou, J.R. Bond, and S.D.M. White, Mon. Not. R. Astron. Soc. 258, 1 (1992).

[17] C. Pryke, N.W. Halverson, E.M. Leitch, J. Kovac, J.E. Carlstrom, W.L. Holzapfel, and M. Dragovan, astro-ph/01004490.

[18] S.M. Carroll, W.H. Press, and E.L. Turner, Annu. Rev. Astron. Astrophys. 30, 499 (1992).

[19] E. Lokas and Y. Hoffman, astro-ph/0108283.

[20] L. Wang and P. Steinhardt, Astrophys. J. 508, 483 (1998).

[21] SNAP Collaboration, P. Nugent, in San Juan 2000, Particle Physics and Cosmology, p. 263 (2000).

[22] F. Adams and G. Laughlin, Rev. Mod. Phys. 69, 337 (1997). 